\title{
Approximation in weighted spaces of vector functions
}

\author{
ILEANA BUCUR AND GAVRIIL PALTINEANU*
}

\begin{abstract}
In this paper, we present the duality theory for general weighted space of vector functions. We mention that a characterization of the dual of a weighted space of vector functions in the particular case $V \subset C^{+}(X)$ is mentioned by J. B. Prolla in [6]. Also, we extend de Branges lemma in this new setting for convex cones of a weighted spaces of vector functions (Theorem 4.2). Using this theorem, we find various approximations results for weighted spaces of vector functions: Theorems 4.2-4.6 as well as Corollary 4.3. We mention also that a brief version of this paper, in the particular case $V \subset C^{+}(X)$, is presented in [3], Chapter 2, subparagraph 2.5.
\end{abstract}

Keywords: Nachbin family, weighted space of vector functions, $p$-Radon measure, polar set, extreme point, convex cone, antialgebraic set with respect to a pair (M, C).

2020 Mathematics Subject Classification: 41A10, 46J10.

Dedicated to Professor Francesco Altomare on the occasion of his 70th birthday.

\section{INTRODUCTION}

The weighted spaces of scalar functions was introduced and studied by L. Nachbin in [4] (see also [5]). We recall that if $V$ is a Nachbin family of upper semi-continuous functions on the locally compact spaces $X$, then the weighted space associated to $V$, denoted by $C V_{0}(X)$, is the set of all continuous functions $f$ on $X$ such that the function $f \cdot v$ vanishes at infinity. Any weight $v \in V$ generate a seminorm $p_{v}: C V_{0}(X) \rightarrow \mathbb{R}_{+}$defined by $p_{v}(f)=\sup \{v(x) \cdot|f(x)|: x \in X\}$. The locally convex topology defined by this family of seminorms is denoted by $\omega_{V}$ and it will be called the weighted topology on $C V_{0}(X)$. For some specific families of weights $V$, some different classes of continuous functions on a locally compact space are obtained, namely the functions with compact support, bounded functions, the functions vanishing at infinity, the rapidly decreasing functions at infinity and so on. A characterization of the dual space of the locally convex spaces $\left(C V_{0}(X), \omega_{V}\right)$ was obtained by W. H. Summers in [7]. More precisely, he showed that if $V \leq C^{+}(X)$ then, the dual space $\left[C V_{0}(X)\right]^{*}$ is isomorphic with the space $V \cdot M_{b}(X)$, where $M_{b}(X)$ is the space of all bounded Radon measure on $X$. A similar result for weighted spaces of vector functions, in the particular case $V \subset C^{+}(X)$, is mentioned by J. B. Prolla in [6]. In Theorem 3.1 of this paper, we obtain a characterization of the dual of a weighted space of vector functions in the general case of the upper semi-continuous weights. The key to getting this result is a new result of Measure Theory, namely Proposition 2.1, in which it is proved that if $U: \mathrm{K}(X, E) \rightarrow \mathbb{R}$ is a $p$-Radon measure, then there exists a smallest

Received: 14.11.2020; Accepted: 22.02.2021; Published Online: 03.03.2021

*Corresponding author: Gavrul Paltineanu; gavriil.paltineanu@gmail.com

DOI: $10.33205 / \mathrm{cma} .825986$ 
positive Radon measure on $X$, denoted by $|U|$, such that

$$
|U(f)| \leq \int p \circ f d|U|, \forall f \in \mathrm{K}(X, E) .
$$

Using two fundamental tools in functional analysis: Hahn-Banach and Krein -Milman theorems, in 1959, Louis de Branges [1] give a nice proof of Stone-Weierstrass theorem on algebras of real continuous functions on a compact Hausdorff space. Some generalizations of de Branges lemma for weighted space of scalar functions was obtained in [2]. In the last part of this paper, we present a generalization of de Branges lemma for a convex cone in a weighted spaces of vector functions (Theorem 4.2). Using this theorem, we obtain various approximations results for weighted spaces of vector functions: Theorems 4.2-4.6 as well as Corollary 4.3.

\section{WEIGHTED SPACES OF VECTOR FUNCTIONS}

Let $X$ be a locally compact Hausdorff space, let $E$ be a locally convex complete space endowed with a family $\mathrm{P}$ of seminorms of $E$. We denote by $C(X, E)$ the set of all continuous functions $f: X \rightarrow E$ and by $C_{0}(X, E)$ respectively $\mathrm{K}(X, E)$, the set of continuous functions vanishing at infinity, respectively having compact support. We recall that a function $f: X \rightarrow E$ vanishes at infinity if $\lim _{x \rightarrow \infty} f(x)=0$, i.e., for any $p \in \mathrm{P}$ and any $\varepsilon>0$, there exists a compact subset $K_{\varepsilon, p}$ of $X$ such that

$$
p[f(x)]<\varepsilon, \forall x \in X \backslash K_{\varepsilon, p} .
$$

Further, we shall denote by $\mathcal{F}_{0}(X, E)$ the set of all functions $f: X \rightarrow E$ vanishing at infinity.

Definition 2.1. A family $V$ of upper semi-continuous, non-negative functions on $X$ such that for any $v_{1}, v_{2} \in V$ and any $\lambda \in \mathbb{R}, \lambda>0$ there exists $w \in V$ such that

$$
v_{i}(x) \leq \lambda \cdot w(x), \forall x \in X, i=1,2
$$

will be called a Nachbin family on $X$. Any element of $V$ will be called a weight.

If $V$ is a Nachbin family of weights on $X$, we denote by

$$
C V_{0}(X, E)=\left\{f \in C(X, E) ; v \cdot f \in C_{0}(X, E), \forall v \in V\right\} .
$$

We endow this linear space with so called the weighted topology $\omega_{V, P}$, given by the family of seminorms $\|\cdot\|_{v, p}$ or $\|\cdot\|_{p_{v}}$ defined by

$$
\|f\|_{p_{v}}=\|f\|_{v, p}=\sup \{v(x) \cdot p[f(x)], \forall x \in X\}, \forall f \in C V_{0}(X, E) .
$$

A base of neighborhoods of the origin in $C V_{0}(X, E)$ is the family $\left(B_{v, p}\right)_{v \in V, p \in \mathrm{P}}$ given by

$$
B_{v, p}=\left\{f \in C V_{0}(X, E) ;\|f\|_{v, p} \leq 1\right\} .
$$

Further, the space $C V_{0}(X, E)$ endowed with the weighted topology $\omega_{V, \mathrm{P}}$ will be called the weighted space of vector functions. As in the scalar case, one can see that $\mathrm{K}(X, E)$ is a dense subset of $C V_{0}(X, E)$ with respect to the weighted topology $\omega_{V, \mathrm{P}}$. For any $p \in \mathrm{P}$ and any $f \in$ $\mathrm{K}(X, E)$, we denote

$$
\|f\|_{p}=\sup _{x \in X} p[f(x)] .
$$

Obviously, $\|f\|_{p}<\infty$ since $p: E \rightarrow \mathbb{R}_{+}$is a continuous function on the locally compact space $E$ and $f(X)=f\left(K_{f}\right) \cup\{0\}$ is a compact subset of $E$, where $K_{f}$ denotes the support of $f$. If we endow $\mathrm{K}(X, E)$ with the family of seminorms $\left(\|\cdot\|_{p}\right)_{p \in \mathrm{P}^{\prime}}$, then $\mathrm{K}(X, E)$ becomes a locally convex space and we shall denote by $\tau_{\mathrm{P}}$ the topology given by these seminorms $\left(\|\cdot\|_{p}\right)_{p \in \mathrm{P}}$ 
Definition 2.2. A linear map $U: \mathrm{K}(X, E) \rightarrow \mathbb{R}$ is called a $p$-Radon measure, where $p \in \mathrm{P}$, if for any compact subset $K \subset X$ there exists a positive number $\alpha_{K}$ such that for any $f \in \mathrm{K}(X, E), f=0$ on $X \backslash K$, we have

$$
|U(f)| \leq \alpha_{K} \cdot\|f\|_{p} .
$$

If $\alpha_{K}$ does not depend of the compact $K$, then $U$ is called a $p$-bounded Radon measure. The smallest $\alpha \in \mathbb{R}_{+}$, such that $|U(f)| \leq \alpha \cdot\|f\|_{p}$ will be denoted by $\|U\|_{p}$.

Proposition 2.1. If $U: \mathrm{K}(X, E) \rightarrow \mathbb{R}$ is a $p$-Radon measure, then there exists a smallest positive Radon measure on $X$, denoted by $|U|$, such that

$$
|U(f)| \leq \int p \circ f d|U|, \forall f \in \mathrm{K}(X, E) .
$$

Moreover, for any function $\varphi \in \mathrm{K}(X, \mathbb{R})$, the map $\varphi U: \mathrm{K}(X, E) \rightarrow \mathbb{R}$ given by

$$
\varphi U(\psi)=U(\varphi \cdot \psi), \forall \psi \in \mathrm{K}(X, E)
$$

is a p-bounded Radon measure and we have

a) $\|\varphi U\|_{p}=|\varphi U|$ (1) and generally $\|U\|_{p}=|U|$ (1) if $U$ is $p$ - bounded,

b) $|\varphi U|=|\varphi| \cdot|U|,\|\varphi U\|_{p}=|\varphi U|(1)=(|\varphi| \cdot|U|)(1)=\int|\varphi| d|U|$.

Proof. Passing to a factorization, we may suppose that $p$ is a norm on $X$. We consider a relatively compact open subset $D$ of the locally compact space $X$ and for any $\varphi \in \mathrm{K}(X, \mathbb{R}), \varphi \geq 0$ and $\operatorname{supp} \varphi \subset D$, we put by definition

$$
|U|(\varphi)=\sup \{U(\psi) ; \psi \in \mathrm{K}(X, E), p \circ \psi \leq \varphi\}=\sup \{|U(\psi)| ; \psi \in \mathrm{K}(X, E), p \circ \psi \leq \varphi\} .
$$

Since $\bar{D}$ is compact and $\psi(x)=0$, if $\varphi(x)=0$, we deduce that $\psi=0$ outside $\bar{D}$ and therefore there exists $\alpha \in \mathbb{R}_{+}$such that $|U(\psi)| \leq \alpha \cdot\|\psi\|_{p} \leq \alpha \cdot\|\varphi\|$, where $\|\varphi\|$ is the uniform norm of $\varphi$ on $X$. Hence $|U|(\varphi) \leq \alpha \cdot\|\varphi\|$ for all $\varphi \in \mathrm{K}(X, \mathbb{R}), \varphi \geq 0$ and $\operatorname{supp} \varphi \subset D$. We show now that for any $\varphi_{i} \in \mathrm{K}(X, \mathbb{R}), \varphi_{i} \geq 0, \operatorname{supp} \varphi_{i} \subset D, i=1,2$, we have

$$
|U|\left(\varphi_{1}+\varphi_{2}\right)=|U|\left(\varphi_{1}\right)+|U|\left(\varphi_{2}\right) .
$$

The inequality $|U|\left(\varphi_{1}+\varphi_{2}\right) \geq|U|\left(\varphi_{1}\right)+|U|\left(\varphi_{2}\right)$ follows just from the definition. Let $\psi \in$ $\mathrm{K}(X, E), p(\psi) \leq \varphi_{1}+\varphi_{2}$. For any $n \in \mathbb{N}^{*}$, we consider the functions $\psi_{i} \in \mathrm{K}(X, E)$ given by

$$
\psi_{i}=\frac{\varphi_{i}}{\varphi_{1}+\varphi_{2}+\frac{1}{n}} \cdot \psi, i=1,2 .
$$

Obviously, we have successively

$$
\begin{gathered}
p\left(\psi_{i}\right)=\varphi_{i} \cdot \frac{p(\psi)}{\varphi_{1}+\varphi_{2}+\frac{1}{n}} \leq \varphi_{i}, i=1,2, \\
\psi-\left(\psi_{1}+\psi_{2}\right)=\frac{1}{n} \cdot \frac{\psi}{\varphi_{1}+\varphi_{2}+\frac{1}{n}}, \\
p\left(\psi-\left(\psi_{1}+\psi_{2}\right)\right) \leq \frac{1}{n} \cdot p\left(\frac{\psi}{\varphi_{1}+\varphi_{2}+\frac{1}{n}}\right), \\
\operatorname{supp}\left(\frac{\psi}{\varphi_{1}+\varphi_{2}+\frac{1}{n}}\right) \subset D, p\left(\frac{\psi}{\varphi_{1}+\varphi_{2}+\frac{1}{n}}\right) \leq 1,\left|U\left(\frac{\psi}{\varphi_{1}+\varphi_{2}+\frac{1}{n}}\right)\right| \leq \alpha, \\
U(\psi) \leq|U|\left(\varphi_{1}\right)+|U|\left(\varphi_{2}\right)+\frac{\alpha}{n}, \forall n \in \mathbb{N}^{*}, \\
U(\psi) \leq|U|\left(\psi_{1}\right)-U\left(\psi_{2}\right)\left|\leq \frac{\alpha}{n}, U\left(\psi_{1}\right)+U\left(\psi_{2}\right)+\frac{\alpha}{n},\left(\varphi_{2}\right),\right| U \mid\left(\varphi_{1}+\varphi_{2}\right)=\sup \left\{U(\psi) ; \psi \in \mathrm{K}(X, E), p(\psi) \leq \varphi_{1}+\varphi_{2}\right\},
\end{gathered}
$$




$$
|U|\left(\varphi_{1}+\varphi_{2}\right) \leq|U|\left(\varphi_{1}\right)+|U|\left(\varphi_{2}\right),|U|\left(\varphi_{1}+\varphi_{2}\right)=|U|\left(\varphi_{1}\right)+|U|\left(\varphi_{2}\right) .
$$

Obviously, we have

$$
|U|(\lambda \cdot \varphi)=\lambda \cdot|U|(\varphi), \forall \lambda \in \mathbb{R}_{+}
$$

and the map $|U|: \mathrm{K}^{+}(X, \mathbb{R}) \rightarrow \mathbb{R}_{+}$is a positive Radon measure on $X$. Just from the definition, we have

$$
|U(\psi)| \leq|U|(p(\psi)), \forall \psi \in \mathrm{K}(X, E) .
$$

On the other hand, taking a positive Radon measure $\mu$ on $X$ such that $|U(\psi)| \leq \int p(\psi) d \mu$ then for any $\varphi \in \mathrm{K}(X, \mathbb{R}), \varphi \geq 0$, we have

$$
\begin{aligned}
& \int \varphi d \mu \geq \int p(\psi) d \mu, \forall \psi \in \mathrm{K}(X, E), p(\psi) \leq \varphi, \\
& \int \varphi d \mu \geq|U(\psi)|, \forall \psi \in \mathrm{K}(X, E), p(\psi) \leq \varphi, \\
& \int \varphi d \mu \geq|U|(\varphi),|U| \leq \mu \text { on } \mathrm{K}^{+}(X, \mathbb{R}) .
\end{aligned}
$$

a) For any $\varphi \in \mathrm{K}(X, \mathbb{R})$, the map $\varphi U: \mathrm{K}(X, E) \rightarrow \mathbb{R}$ defined by $\varphi U(\psi)=U(\varphi \cdot \psi)$ is linear and we have

$$
|\varphi U(\psi)| \leq \alpha_{K} \cdot\|\varphi \cdot \psi\|_{p} \leq \alpha_{K} \cdot\|\varphi\| \cdot\|\psi\|_{p},
$$

where $K=\operatorname{supp} \varphi$ and therefore $\varphi U$ is a $p$ - bounded Radon measure on $\mathrm{K}(X, E)$. Further, we have

$$
\begin{aligned}
|\varphi U|(1) & =\int 1 d|\varphi U| \\
& =\sup \left\{\int h d|\varphi U| ; 0 \leq h \leq 1, h \in \mathrm{K}(X, \mathbb{R})\right\} \\
& =\sup \{(\varphi U)(\psi) ; \psi \in \mathrm{K}(X, \mathbb{R}), p(\psi) \leq 1\} \\
& =\|\varphi U\|_{p}
\end{aligned}
$$

(In fact, for any $p$ - bounded Radon measure $U^{\prime}: \mathrm{K}(X, E) \rightarrow \mathbb{R}$ we have, using the definition of $\left|U^{\prime}\right|$ :

$$
\left.\left\|U^{\prime}\right\|_{p}=\left|U^{\prime}\right|(1)=\int_{X} d\left|U^{\prime}\right|\right),
$$

b) The inequality $|\varphi U| \leq|\varphi| \cdot|U|$ follows immediately. Indeed, if $h \in \mathrm{K}(X, \mathbb{R}), h \geq 0$ then,

$$
\begin{aligned}
|\varphi U|(h) & =\sup \{U(\varphi \cdot \psi) ; p(\psi) \leq h\} \\
& \leq \sup \{|U|(p(\varphi \cdot \psi) ; p(\psi) \leq h\} \\
& =\sup \{(|\varphi| \cdot|U|)(p(\psi)) ; p(\psi) \leq h\} \\
& =(|\varphi| \cdot|U|)(h) .
\end{aligned}
$$

Hence $|\varphi U|(h) \leq|\varphi| \cdot|U|(h)$ for any $h \in \mathrm{K}(X, \mathbb{R}), h \geq 0$. For the converse inequality, we restrict ourself to the case $\varphi \geq 0$. Let us consider $\psi \in \mathrm{K}(X, E)$ such that $p(\psi) \leq h \cdot \varphi$ and for any $n \in \mathbb{N}^{*}$, we consider the function $f_{n} \in \mathrm{K}(X, E)$ defined by

$$
f_{n}=\frac{\psi}{\varphi+\frac{1}{n}} \text {. }
$$

Obviously, $p\left(f_{n}\right) \leq h$ and therefore

$$
|\varphi U|(h) \geq U\left(\varphi \cdot f_{n}\right), p\left(\varphi \cdot f_{n}\right) \leq h \cdot \varphi, p\left(\psi-\varphi \cdot f_{n}\right) \leq \frac{1}{n} \cdot p(h) .
$$


Since $\psi=0$ outside $K=\operatorname{supp} \varphi$, we have

$$
\psi-\varphi \cdot f_{n}=0 \text { on } X \backslash K, p\left(\psi-\varphi \cdot f_{n}\right) \leq \frac{1}{n} \cdot\|h\|,\left|U\left(\psi-\varphi \cdot f_{n}\right)\right| \leq \alpha_{K} \cdot \frac{1}{n} \cdot\|h\|
$$

and therefore

$$
|\varphi U|(h) \geq U\left(\varphi \cdot f_{n}\right) \geq U(\psi)-\alpha_{K} \cdot\|h\| \cdot \frac{1}{n},|\varphi U|(h) \geq U(\psi) .
$$

But

$$
(\varphi|U|)(h)=|U|(\varphi \cdot h)=\sup \{U(\psi) ; \psi \in \mathrm{K}(X, E), p(\psi) \leq h \cdot \varphi\} .
$$

From the preceding two lines, we get $|\varphi U|(h) \geq(\varphi|U|)(h)$ and finally $|\varphi U|=|\varphi| \cdot|U|$.

Proposition 2.2. Let $U: \mathrm{K}(X, E) \rightarrow E$ be a $p$-Radonn measure, $f: X \rightarrow \overline{\mathbb{R}}$ be an integrable function with respect to the positive Radon measure $|U|$ (i.e., $f \in \mathrm{L}^{1}(|U|)$ ) and let $\left(\varphi_{n}\right)_{n}$ be a sequence in $\mathrm{K}(X, \mathbb{R})$ such that $\lim _{n \rightarrow \infty} \varphi_{n}(x)=f(x),|U|-$ a.e. on $X$ and such that

$$
\lim _{n \rightarrow \infty} \int\left|f-\varphi_{n}\right| d|U|=0 .
$$

Then, the sequence of $p-$ bounded Radon measures $\left(\varphi_{n} U\right)_{n}$ is convergent to a $p$ - bounded Radon measure (depending of $f$ only), denoted by $f U$, i.e., $\lim _{n \rightarrow \infty}\left\|f U-\varphi_{n} U\right\|_{p}=0$. Moreover, we have

$$
|f U|=|f| \cdot|U| \text {. }
$$

Proof. Since $\lim _{n \rightarrow \infty} \int\left|f-\varphi_{n}\right| d|U|=0$, we deduce that $\lim _{n, m \rightarrow \infty} \int\left|\varphi_{n}-\varphi_{m}\right| d|U|=0$ and therefore, using Proposition 2.1, we have

$$
\lim _{n, m \rightarrow \infty}\left\|\varphi_{n} U-\varphi_{m} U\right\|_{p}=\lim _{n, m \rightarrow \infty} \int\left|\varphi_{n}-\varphi_{m}\right| d|U|=0 .
$$

Hence for any $\psi \in \mathrm{K}(X, E)$, the sequence $\left(\varphi_{n} U(\psi)\right)_{n}$ of real numbers is convergent to a number denoted $f U(\psi)$ and for any $\varepsilon>0$, there exists $n_{\varepsilon} \in \mathbb{N}^{*}$ such that

$$
\begin{gathered}
\left|\varphi_{n} U(\psi)-\varphi_{m} U(\psi)\right| \leq\left\|\varphi_{n} U-\varphi_{m} U\right\|_{p} \cdot\|\psi\|_{p} \leq \varepsilon \cdot\|\psi\|_{p}, \forall n, m \geq n_{\varepsilon}, \\
\left|f U(\psi)-\varphi_{m} U(\psi)\right| \leq \varepsilon \cdot\|\psi\|_{p}, \forall m \geq n_{\varepsilon}, \\
|f U(\psi)| \leq\left|\varphi_{m} U(\psi)\right|+\varepsilon \cdot\|\psi\|_{p} \leq\left(\left\|\varphi_{m} U\right\|_{p}+\varepsilon\right) \cdot\|\psi\|_{p} .
\end{gathered}
$$

Hence $f U$ is a $p$ - bounded Radon measure on $\mathrm{K}(X, E), \lim _{m \rightarrow \infty}\left\|f U-\varphi_{m} U\right\|_{p}=0$ (Particularly if $f=0|U|$ a.e., from the relation $\lim _{n \rightarrow \infty} \int\left|f-\varphi_{n}\right| d|U|=0$, we deduce $\lim _{n \rightarrow \infty} \int\left|\varphi_{n}\right| d|U|=0$ and therefore $\lim _{n \rightarrow \infty}\left\|\varphi_{n} U\right\|_{p}=\lim _{n \rightarrow \infty} \int\left|\varphi_{n}\right| d|U|=0, \lim _{n \rightarrow \infty}\left(\varphi_{n} U\right)(\psi)=0, \forall \psi \in \mathrm{K}(X, E)$. This shows that the element $f U$, previously defined, depends only on $f$, does not depend on the choice of the sequence $\left(\varphi_{n}\right)_{n}$ tending to $\left.f\right)$. Let now $h \in \mathrm{K}(X, \mathbb{R}), 0 \leq h \leq 1$ and let $\psi \in \mathrm{K}(X, E)$ be such that $p(\psi) \leq h$. We have

$$
\begin{gathered}
\left|f U(\psi)-\varphi_{n} U(\psi)\right| \leq\left\|f U-\varphi_{n} U\right\|_{p} \cdot\|\psi\|_{p} \leq\left\|f U-\varphi_{n} U\right\|, \forall n \in \mathrm{N}, \\
\left(\varphi_{n} U\right)(\psi)-\left\|f U-\varphi_{n} U\right\|_{p} \leq f U(\psi) \leq \varphi_{n} U(\psi)+\left\|f U-\varphi_{n} U\right\|_{p}, \\
\left|\varphi_{n} U\right|(h)-\left\|f U-\varphi_{n} U\right\|_{p} \leq|f U|(h) \leq\left|\varphi_{n} U\right|(h)+\left\|f U-\varphi_{n} U\right\|_{p} .
\end{gathered}
$$

Using Proposition $2.1 \mathrm{~b}$ ), we deduce that

$$
\left|\varphi_{n}\right| \cdot|U|(h)-\left\|f U-\varphi_{n} U\right\|_{p} \leq|f U|(h) \leq\left|\varphi_{n}\right| \cdot|U|(h)+\left\|f U-\varphi_{n} U\right\|_{p}
$$




$$
\int\left|\varphi_{n}\right| \cdot h d|U|-\left\|f U-\varphi_{n} U\right\|_{p} \leq|f U|(h) \leq \int\left|\varphi_{n}\right| \cdot h d|U|+\left\|f U-\varphi_{n} U\right\|_{p} .
$$

Passing to the limit on $n$, we get

$$
\begin{aligned}
& \int|f| \cdot h d|U| \leq|f U|(h) \leq \int|f| \cdot h d|U|, \\
& |f U|(h)=\int|f| \cdot h d|U|=|f| \cdot|U|(h) .
\end{aligned}
$$

The last equality holds for $0 \leq h \leq 1$ and therefore for all $h \in \mathrm{K}(X, \mathbb{R}), h \geq 0$, i.e.,

$$
|f U|=|f| \cdot|U| \text {. }
$$

\section{ON THE DUAL OF WEIGHTED SPACES OF VECTOR FUNCTIONS}

Let $E, \mathrm{P}, X$ and $V$ as in the preceding section. For any $p \in \mathrm{P}$ and $v \in V$, let

$$
B_{v, p}=\left\{f \in C V_{0}(X, E) ; p_{v}(f) \leq 1\right\},
$$

where $p_{v}(f)=\sup \{v(x) \cdot p[f(x)] ; \forall x \in X\}=\|f\|_{v, p}, \forall f \in C V_{0}(X, E)$. The linear vector space $C V_{0}(X, E)$ endowed with the family $\left(p_{v}\right)_{p \in \mathrm{P}, v \in V}$ of seminorms is a locally convex space whose fundamental system of neighborhoods of the origin is just the family $\left(B_{v, p}\right)_{v \in V, p \in \mathrm{P}}$. We recall that we have denoted by $\omega_{V, \mathrm{P}}$ the weighted topology on $C V_{0}(X, E)$ given by the family of seminorms $\left(p_{v}\right)_{p \in \mathrm{P}, v \in V}$. It is no lost of generality if we suppose that for any real number $\alpha, \alpha>0$, we have $\alpha \cdot p \in \mathrm{P}, \alpha \cdot v \in V$ for any $p \in \mathrm{P}$ and any $v \in V$. So the dual of the locally convex space $\left(C V_{0}(X, E), \omega_{V, \mathrm{P}}\right)$ is the set $\bigcup_{v \in V, p \in \mathrm{P}} B_{v, p}^{0}$, where

$$
B_{v, p}^{0}=\left\{T: C V_{0}(X, E) \rightarrow \mathbb{R} ; T \text { linear, } T(f) \leq 1, \forall f \in B_{v, p}\right\} .
$$

If we denote by $\left[C V_{0}(X, E)\right]^{*}$ this dual, then for any subset $M$ of $C V_{0}(X, E)$ (respectively of $\left.\left[C V_{0}(X, E)\right]^{*}\right)$, we denote by $M^{0}$ the polar of $M$ i.e.,

$$
M^{0}=\left\{T \in\left[C V_{0}(X, E)\right]^{*} ; T(m) \leq 1, \forall m \in M\right\}
$$

respectively

$$
M^{0}=\left\{f \in C V_{0}(X, E) ; m(f) \leq 1, \forall m \in M\right\} .
$$

The map on $C V_{0}(X, E) \times\left[C V_{0}(X, E)\right]^{*} \rightarrow \mathbb{R},(f, T) \rightarrow\langle f, T\rangle=T(f)$ is a natural duality between the linear space $C V_{0}(X, E)$ and $\left[C V_{0}(X, E)\right]^{*}$. The smallest topology on $\left[C V_{0}(X, E)\right]^{*}$ making continuous the maps

$$
T \rightarrow\langle f, T\rangle:\left[C V_{0}(X, E)\right]^{*} \rightarrow \mathbb{R}, \forall f \in C V_{0}(X, \mathbb{R})
$$

is the weak topology on $\left[C V_{0}(X, E)\right]^{*}$. It is known (Alaoglu's Theorem) that for any $(p, v) \in \mathrm{P} \times$ $V$, the set $B_{p, v}^{0}$ is a weakly compact subset of $\left[C V_{0}(X, E)\right]^{*}$. We know also that the topological space $\left[C V_{0}(X, E)\right]^{*}$ is a Hausdorff one with respect to this weak topology. Moreover, since $\mathrm{K}(X, E)$ is a dense subset of $C V_{0}(X, E)$ with respect to the weighted topology $\omega_{V, \mathrm{P}}$, we deduce that

1) any continuous linear functional $L: C V_{0}(X, E) \rightarrow \mathbb{R}$ is completely determined by its restriction to $\mathrm{K}(X, E)$,

2) the smallest topology on $\left[C V_{0}(X, E)\right]^{*}$ making continuous all linear functionals

$$
T \rightarrow\langle f, T\rangle:\left[C V_{0}(X, E)\right]^{*} \rightarrow \mathbb{R}, \forall f \in \mathrm{K}(X, \mathbb{R})
$$

is also a Hausdorff one and therefore its restriction to $B_{p, v}^{0}$ coincides with the restriction to $B_{p, v}^{0}$ of the weak topology on $\left[C V_{0}(X, E)\right]^{*}$. 
We conclude that any element of the dual of the locally convex space $\left(\mathrm{K}(X, E), \omega_{V, \mathrm{P}} \mid \mathrm{K}(X, E)\right)$ may be uniquely extended to an element of $\left[C V_{0}(X, E)\right]^{*}$. The following assertion characterizes the elements of $\left[C V_{0}(X, E)\right]^{*}$ in terms of Radon measures on $\mathrm{K}(X, E)$. With the above notations, we have

Theorem 3.1. For any $(p, v) \in \mathrm{P} \times V$, we have

a) The restriction of any element $T \in B_{p, v}^{0}$ to $\mathrm{K}(X, E)$ is a $p$-Radon measure on $\mathrm{K}(X, E)$ such that the function $\frac{1}{v}$ is integrable with respect to the positive Radon measure $|T|$ on $X$.

Moreover, the following relation holds:

$$
\int \frac{1}{v} d|T|=\|T\|_{p, v}=\sup \left\{T(f) ; f \in B_{p, v}\right\},
$$

b) For any $p$-Radon measure $U$ on $\mathrm{K}(X, E)$ such that the function $\frac{1}{v}$ is $|U|$ - integrable, there exists $T \in B_{p, v}^{0}$ such that $U$ is the restriction of $T$ to $\mathrm{K}(X, E)$.

Proof. a) Let $T \in B_{p, v}^{0}$ and let $K$ be a compact subset of $X$. Since $v: X \rightarrow[0, \infty)$ is an upper semi-continuous function, its upper bound $\alpha_{K}$ on $K$ is finite. Let $\varphi \in \mathrm{K}(X, E)$ such that $\varphi=0$ on $X \backslash K$. We have

$$
\begin{gathered}
\sup \{v(x) \cdot p(\varphi(x)): x \in X\} \leq \alpha_{K} \cdot \sup \{p(\varphi(x)): x \in X\}=\alpha_{K} \cdot\|\varphi\|_{p}, \\
\frac{\varphi}{\alpha_{K} \cdot\|\varphi\|_{p}} \in B_{p, v},\left|T\left(\frac{\varphi}{\alpha_{K} \cdot\|\varphi\|_{p}}\right)\right| \leq 1,|T(\varphi)| \leq \alpha_{K} \cdot\|\varphi\|_{p},
\end{gathered}
$$

i.e., the restriction of $T$ to $\mathrm{K}(X, E)$, denoted also by $T$, is a $p$-Radon measure. We have

$$
\begin{aligned}
\|T\|_{p, v} & =\sup \left\{T(f), f \in C V_{0}(X, E), p_{v}(f) \leq 1\right\} \\
& =\sup \left\{T(f), f \in \mathrm{K}(X, E), p_{v}(f) \leq 1\right\} \\
& =\sup \left\{T(f), f \in \mathrm{K}(X, E), p(f) \leq \frac{1}{v}\right\} \\
& =\int \frac{1}{v} d|T| .
\end{aligned}
$$

b) Let $U$ be a $p$-Radon measure on $\mathrm{K}(X, E)$ such that the function $\frac{1}{v}$ is $|U|$-integrable. Then, we have

$$
\begin{aligned}
\infty>\int \frac{1}{v} d|U| & =\sup \left\{\int \varphi d|U| ; \varphi \in \mathrm{K}(X, \mathbb{R}), 0 \leq \varphi \leq \frac{1}{v}\right\} \\
& =\sup _{\varphi \leq \frac{1}{v}}\{U(\psi) ; \psi \in \mathrm{K}(X, E), p(\psi) \leq \varphi\} \\
& =\sup \left\{U(\psi) ; \psi \in \mathrm{K}(X, E), p(\psi) \leq \frac{1}{v}\right\} \\
& =\sup \{U(\psi) ; \psi \in \mathrm{K}(X, E), v(x) \cdot p(\varphi(x)) \leq 1\} \\
& =\|U\|_{p, v} .
\end{aligned}
$$

Remark 3.1. From the above considerations, we deduce that:

The elements $T \in B_{p, v}^{0}$ are $p$-Radon measure on $\mathrm{K}(X, E)$ such that the function $\frac{1}{v}$ is $|T|$ - integrable and $\|T\|_{p, v}=\int \frac{1}{v} d|T| \leq 1$. 
Proposition 3.3. Let $T$ be a $p$ - Radon measure, $T \in B_{p, v}^{0}$. If $f \in C V_{0}(X, E)$, then

$$
|T(f)| \leq \int p(f) d|T|
$$

Proof. Let $\left(\psi_{n}\right)_{n}$ be a sequence in $\mathrm{K}(X, E)$ such that $\lim _{n \rightarrow \infty}\left\|f-\psi_{n}\right\|_{p, v}=0$. We know that $\left|T\left(\psi_{n}\right)\right| \leq \int p\left(\psi_{n}\right) d|T|$ and $T(f)=\lim _{n \rightarrow \infty} T\left(\psi_{n}\right)$. On the other hand

$$
\begin{aligned}
p\left(f-\psi_{n}\right) & \leq \frac{\left\|f-\psi_{n}\right\|_{p, v}}{v} \text { on } X \\
\int p\left(f-\psi_{n}\right) d|T| & \leq\left\|f-\psi_{n}\right\|_{p, v} \cdot \int \frac{1}{v} d|T| \leq\left\|f-\psi_{n}\right\|_{p, v} \\
\int\left|p(f)-p\left(\psi_{n}\right)\right| d|T| & \leq \int p\left(f-\psi_{n}\right) d|T| \leq\left\|f-\psi_{n}\right\|_{p, v}, \\
\int p(f) d|T| & =\lim _{n \rightarrow \infty} \int p\left(\psi_{n}\right) d|T| .
\end{aligned}
$$

Hence

$$
|T(f)|=\lim _{n \rightarrow \infty}\left|T\left(\psi_{n}\right)\right| \leq \lim _{n \rightarrow \infty} \int p\left(\psi_{n}\right) d|T|=\int p(f) d|T| .
$$

Corollary 3.1. If $T \in B_{p, v}^{0}$ and $f \in C V_{0}(X, E)$ is such that $f=0$ on $\operatorname{supp}|T|$, then $T(f)=0$.

\section{LEMMA DE BRANGES AND APPROXIMATION RESULTS}

In this section, we preserve all notations used in the preceding paragraphs. For any subset $A \subset C V_{0}(X, E)$, we denote by $A^{0}$ the polar of $A$, i.e.,

$$
A^{0}=\left\{T \in\left[C V_{0}(X, E)\right]^{*} ; T(a) \leq 1, \forall a \in A\right\} .
$$

If $C$ is a convex cone of the real vector space $C V_{0}(X, E)$ then, one can see that

$$
\mathrm{C}^{0}=\left\{T \in\left[C V_{0}(X, E)\right]^{*} ; T(c) \leq 0, \forall c \in \mathrm{C}\right\} .
$$

Theorem 4.2. Let $\mathrm{C}$ be a convex cone in $C V_{0}(X, E), p \in \mathrm{P}, v \in V$ and let $L \in B_{p, v}^{0} \cap \mathrm{C}^{0}, L \neq 0$ be an extreme point of the convex and compact subset $B_{p, v}^{0} \cap \mathrm{C}^{0}$. If $h \in C(X,[0,1])$ is such for any $c \in \mathrm{C}$, we have $h \cdot c|\sigma(|L|) \in \mathrm{C}| \sigma(|L|)$ and $(1-h) \cdot c|\sigma(|L|) \in \mathrm{C}| \sigma(|L|)$, then $h$ is constant on $\sigma(|L|)-$ the support of the positive Radon measure $|L|$ on $X$.

Proof. Since $L \neq 0$ and $L$ is an extreme point of the subset $B_{p, v}^{0} \cap \mathrm{C}^{0}$, we have $\|L\|_{p, v}=\int \frac{1}{v} d|L|$. If $h$ is an arbitrary element in $C(X,[0,1])$, then the map $h L: \mathrm{K}(X, E) \rightarrow \mathbb{R}$, given by $h L(\psi)=$ $L(h \cdot \psi)$, is a $p$-Radon measure on $\mathrm{K}(X, E)$. It is not so difficult to show, using the definition, that $|h L|=|h| \cdot|L|$. Obviously, the function $\frac{1}{v}$ is $|h| \cdot|L|-$ integrable and using Remark 3.1 and the relations

$$
\|h L\|_{p, v}=\int \frac{1}{v} d|h L|=\int \frac{h}{v} d|L| \leq \int \frac{1}{v} d|L| \leq 1,
$$

we get $h L \in B_{p, v}^{0}$. Analogously, the map $(1-h) L: \mathrm{K}(X, E) \rightarrow \mathbb{R}$ given by $(1-h) L(\psi)=$ $L((1-h) \cdot(\psi))$ is a $p$-Radon measure and

$$
\|(1-h) L\|_{p, v}=\int \frac{1-h}{v} d|L| \leq \int \frac{1}{v} d|L|=1,(1-h) L \in B_{p, v}^{0} .
$$

If we denote $\alpha=\|h L\|_{p, v}=\int \frac{h}{v} d|L|, \beta=\|(1-h) L\|_{p, v}=\int \frac{1-h}{v} d|L|$, we have $\alpha+\beta=$ $\int \frac{1}{v} d|L|=1$. We remark also that the function $\frac{1}{v}$ is strictly positive on $X$. If $\alpha=0$, then 
$h=0 \quad|L|$ a.e. on $\sigma(|L|)$. Since the function $h$ is continuous, it results that $h=0$ on $\sigma(|L|)$, i.e., $h$ is constant on $\sigma(|L|)$. Analogously, if $\beta=0$, we obtain $h=1$ on $\sigma(|L|)$, i.e., $h$ is constant on $\sigma(|L|)$. We suppose further $\alpha \neq 0, \beta \neq 0$ and we denote

$$
L_{1}=\frac{1}{\alpha} \cdot h L, L_{2}=\frac{1}{\beta} \cdot(1-h) L .
$$

Obviously, $\left\|L_{i}\right\|_{p, v}=1, i=1,2$ and $\alpha \cdot L_{1}+\beta \cdot L_{2}=L$. We show now that $L_{i} \in \mathrm{C}^{0}, i=1,2$, if for any $c \in \mathrm{C}$ there exist $c_{1}, c_{2} \in \mathrm{C}$ such that $h \cdot c=c_{1},(1-h) \cdot c=c_{2}$ on $\sigma(|L|)$. Since the functions $h \cdot c,(1-h) \cdot c, c_{1}, c_{2}$ belong to $C V_{0}(X, E)$ and $h \cdot c=c_{1}$ on $\sigma(|L|)$, respectively $(1-h) \cdot c=c_{2}$ on $\sigma(|L|)$, using Corollary 3.1, we get

$$
\begin{gathered}
L(h \cdot c)=L\left(c_{1}\right) \leq 0, L((1-h) \cdot c)=L\left(c_{2}\right) \leq 0 \\
L_{1}(c)=\frac{1}{\alpha} \cdot L(h \cdot c)=\frac{1}{\alpha} \cdot L\left(c_{1}\right) \leq 0, L_{2}(c)=\frac{1}{\beta} \cdot L((1-h) \cdot c)=\frac{1}{\beta} \cdot L\left(c_{2}\right) \leq 0 .
\end{gathered}
$$

Hence $L_{1}, L_{2}$ belong to the set $B_{p, v}^{0} \cap C^{0}$ and since $L=\alpha \cdot L_{1}+\beta \cdot L_{2}$, we get $L_{1}=L_{2}=L$. Hence $\left|L_{1}\right|=|L|$, i.e., the measures $\frac{h}{\alpha} \cdot|L|$ and $|L|$ coincide and therefore $\frac{h}{\alpha}=1$ almost everywhere on $\sigma(|L|)$. But $h$ is continuous and hence $h=\alpha$ on $\sigma(|L|)$.

Definition 4.3. A subset $\mathrm{M} \subset C(X,[0,1])$ is called complemented, if for any $h \in \mathrm{M}$, the function $1-h$ belongs to $\mathrm{M}$. If $\mathrm{C} \subset C V_{0}(X, E)$ is a convex cone and $\mathrm{M} \subset C(X,[0,1])$ is a complemented family, then a subset $S \subset X$ is called antialgebraic with respect to the pair $(\mathrm{M}, \mathrm{C})$ (or simpler $(\mathrm{M}, \mathrm{C})-$ antialgebraic), if any $h \in \mathrm{M}$ such that the restriction to $S$ of the functions $h \cdot c$ and $(1-h) \cdot c$ belong to the restriction of $\mathrm{C}$ to $S$ (i.e., $h \cdot c|S \in \mathrm{C}| S,(1-h) \cdot c|S \in \mathrm{C}| S$ ) for any $c \in \mathrm{C}$, is a constant function on $S$.

We can reformulate de Branges lemma (Theorem 4.2) as follows:

Corollary 4.2. For any extreme point $L$ of $B_{p, v}^{0} \cap C^{0}$, the support $\sigma(|L|)$ of the positive Radon measure $|L|$ on $X$ is an antialgebraic subset with respect to the pair $(C(X,[0,1]), C)$. Further, we denote by $\mathrm{S}$ the family of all subsets of $X$ antialgebraic with respect to the pair $(\mathrm{M}, \mathrm{C})$.

The following assertions are almost obvious.

i) $\{x\} \in \mathrm{S}, \forall x \in X$,

ii) $S_{1}, S_{2} \in \mathrm{S}, S_{1} \cap S_{2} \neq \phi \Rightarrow S_{1} \cup S_{2} \in \mathrm{S}$,

iii) $S \in \mathrm{S} \Rightarrow \bar{S} \in \mathrm{S}$,

iv) For any upper directed family $\left(S_{\alpha}\right)_{\alpha \in I}$ from $\mathrm{S}$, we have $\bigcup_{\alpha \in I} S_{\alpha} \in \mathrm{S}$.

If for any $x \in X$, we denote by $S_{x}=\cup\{S ; S \in \mathrm{S}, x \in S\}$, then we have

$$
S_{x}=\overline{S_{x}} \in \mathrm{S}, S_{x} \cap S_{y}=\phi \text { if } S_{x} \neq S_{y} .
$$

The family $\left(S_{x}\right)_{x \in X}$ is a partition of $X$ and for any $S \in \mathrm{S}$ there exists $x \in X$ such that $S \subset S_{x}$. For the general theory of duality, we have for any convex cone $\mathrm{C}, \mathrm{C} \subset C V_{0}(X, E)$, the closure $\overline{\mathrm{C}}$ in $C V_{0}(X, E)$ with respect to the weighted topology $\omega_{\mathrm{P}, V}$ coincides with the bipolar of $\mathrm{C}$ i.e., $\overline{\mathrm{C}}=\mathrm{C}^{00}$. In the our special case, we have the following general approximation theorem.

Theorem 4.3. If $\mathrm{C} \subset C V_{0}(X, E)$ is a convex cone, then the closure of $\mathrm{C}$ in $\left(C V_{0}(X, E), \omega_{\mathrm{P}, V}\right)$ is given by

$$
\overline{\mathrm{C}}=\left\{f \in C V_{0}(X, E) ; f \mid \sigma(|L|) \in \overline{\mathrm{C} \mid \sigma(|L|)}, \forall L \in \operatorname{Ext}\left(B_{p, v}^{0} \cap \mathrm{C}^{0}\right), \forall v \in V, \forall p \in \mathrm{P}\right\} .
$$


Proof. We show only that for any function $g \in C V_{0}(X, E) \backslash \overline{\mathrm{C}}$ there exist $p \in \mathrm{P}, v \in V$ and $L \in \operatorname{Ext}\left(B_{p, v}^{0} \cap \mathrm{C}^{0}\right)$ such that $g \mid \sigma(|L|) \notin \overline{\mathrm{C} \mid \sigma(\mu)}$. Indeed, using Hahn-Banach separation theorem, there exists $T \in\left[C V_{0}(X, E)\right]^{*}$ such that $T \in \mathrm{C}^{0}$ and $T(g)>0$. Let $p \in \mathrm{P}$ and $v \in V$ be such that $|T(f)| \leq\|f\|_{p, v}, \forall f \in C V_{0}(X, E)$ i.e., $|T|\left(\frac{1}{v}\right) \leq 1$. Hence $T \in B_{p, v}^{0} \cap \mathrm{C}^{0}$. Since $B_{p, v}^{0} \cap \mathrm{C}^{0}$ is a compact convex subset of $\left[C V_{0}(X, E)\right]^{*}$ with respect to the weak topology and $T(g)>0$, it follows from Krein-Milman theorem that there exists $L \in \operatorname{Ext}\left(B_{p, v}^{0} \cap \mathrm{C}^{0}\right)$ such that $L(g)>0$. Since $L \in \mathrm{C}^{0}$, we deduce that $\int \varphi d|L| \leq 0$ for any $\varphi \in \overline{\mathrm{C} \mid \sigma(|L|)}$. Hence $g \mid \sigma(|L|) \notin \overline{\mathrm{C} \mid \sigma(|L|)}$.

Let now $\mathrm{M} \subset C(X,[0,1])$ be a complemented family and for any $x \in X$ let $S_{x}$ be the greatest $(\mathrm{M}, \mathrm{C})$ - antialgebraic subset of $X$ containing $x$.

Theorem 4.4. If $\mathrm{C} \subset C V_{0}(X, E)$ is a convex cone, then the closure of $\mathrm{C}$ in $\left(C V_{0}(X, E), \omega_{\mathrm{P}, V}\right)$ is given by

$$
\overline{\mathrm{C}}=\left\{f \in C V_{0}(X, E) ; f \mid S_{x} \in \overline{\mathrm{C} \mid S_{x}}, \forall x \in X\right\} .
$$

Proof. For any $p \in \mathrm{P}, v \in V$ and any extreme point $L$ of the compact convex subset $B_{p, v}^{0} \cap \mathrm{C}^{0}$, the support $\sigma(|L|)$ is a $(\mathrm{M}, \mathrm{C})$ - antialgebraic subset of $X$. If we choose a point $x \in \sigma(|L|)$, then $\sigma(|L|) \subset S_{x}$, and therefore if $f \mid S_{x} \in \overline{\mathrm{C} \mid S_{x}}$, we have also $f \mid \sigma(L) \in \overline{\mathrm{C} \mid \sigma(L)}$. Further, we may use Theorem 4.3.

Theorem 4.5. If $\mathrm{M} \subset C(X,[0,1])$ is a complemented family and the convex cone $\mathrm{C} \subset C V_{0}(X, E)$ is stable with respect to the multiplication of $\mathrm{M}$ (i.e., $c \cdot m \in \mathrm{C}, \forall c \in \mathrm{C}, m \in \mathrm{M}$ ), then we have

$$
\overline{\mathrm{C}}=\left\{f \in C V_{0}(X, E) ; f \mid[x]_{\mathrm{M}} \in \overline{\mathrm{C} \mid[x]_{\mathrm{M}}}, \forall x \in X\right\},
$$

where for any $x \in X$ we denote $[x]_{\mathrm{M}}=\{y \in X ; m(y)=m(x), \forall m \in \mathrm{M}\}$.

Proof. Using just the definitions and previous notations, we deduce that for any $x \in X$ we have $[x]_{\mathrm{M}}=S_{x}$. Further, we use Theorem 4.4.

The following assertion needs to define so called "section in C" by the points of $X$, namely to consider the following convex cone $\mathrm{C}(x)$ in $E$ given by

$$
\mathrm{C}(x)=\{c(x) ; c \in \mathrm{C}\}
$$

and also its closure $\overline{\mathrm{C}(x)}$ in $E$. Certainly the starting convex cone $\mathrm{C}$ in $C V_{0}(X, E)$ may be a linear subspace and in this case $\mathrm{C}(x)$ is a linear subspace in $E$.

Theorem 4.6. If $\mathrm{M} \subset C(X,[0,1])$ is a complemented family and the convex cone $\mathrm{C} \subset C V_{0}(X, E)$ is stable with respect to the multiplication with elements of $\mathrm{M}$ and $\mathrm{M}$ separates the points of $X$, i.e., for any $x, y \in X$ there exists $m \in \mathrm{M}$ such that $m(x) \neq m(y)$, then we have

$$
\overline{\mathrm{C}}=\left\{f \in C V_{0}(X, E) ; f(x) \in \overline{\mathrm{C}(x)}, \forall x \in X\right\} .
$$

Indeed, in this case, for any $x \in X$, we have $[x]_{\mathrm{M}}=\{x\}$ and we close the proof applying Theorem 4.5.

Corollary 4.3. If $\mathrm{M} \subset C(X,[0,1])$ is a complemented family, separating the points of $X$ and $\mathrm{W} \subset$ $C V_{0}(X, E)$ is a linear subspace which is stable with respect to the multiplication with elements of $\mathrm{M}$ and for any $x \in X$ the section $\mathrm{W}(x)$ is a dense subset of the locally convex space $(E, \mathrm{P})$, then

$$
\overline{\mathrm{W}}=C V_{0}(X, E) .
$$


Remark 4.2. For the scalar case $E=\mathbb{R}$, the density of $\mathrm{W}(x)$ in $\mathbb{R}$ is automatically fulfilled unless the case where $\mathrm{W}(x)=\{0\}$ for the points $x$ of a closed subset $F \subset X$. In this case, we have

$$
\overline{\mathrm{W}}=\left\{f \in C V_{0}(X) ; f=0 \text { on } F\right\} .
$$

Even this assertion may be drown from Theorem 4.6 as a particular case where there exists $F \subset X$ such that the section of $\mathrm{C}$ by $x$ is trivial for all $x \in F$ i.e., $\mathrm{C}(x)=\left\{0_{E}\right\}, \forall x \in F$. Anyway Theorem 4.6 may be used in different manners to obtain density results.

\section{REFERENCES}

[1] L. De Branges: The Stone-Weierstrass theorem, Proc. Amer. Math. Soc., 10 (5) (1959), 822-824.

[2] I. Bucur, G. Păltineanu: De Branges type lemma and approximation in weighted spaces, Mediterranean J. Math., (to appear).

[3] I. Bucur, G. Păltineanu: Topics in the uniform approximation of continuous functions, Birkhauser (2020).

[4] L. Nachbin: Weigthed approximation for algebras and modules of continuous functions: real and self-adjoint complex cases, Ann. of Math., 81 (1965), 289-302.

[5] L. Nachbin: Elements of approximation theory, D. Van Nostrand, Princeton (1967).

[6] J. B. Prolla: Bishop's generalized Stone-Weierstrass theorem for weighted spaces, Math. Anal., 191 (4) (1971), 283-289.

[7] W. H. Summers: Dual spaces of weighted spaces, Trans. Amer. Math. Soc., 151 (1) (1970), 323-333.

ILEANA BUCUR

TECHNICAL UNIVERSITY OF CIVIL ENGINEERING BUCHAREST

DepartMENT OF MATHEMATICS AND COMPUTER SCIENCE

BD. LACUL TEI 124, SECTOR 2, 38RO-020396 BUCHAREST, ROMANIA

ORCID: 0000-0001-7832-7087

E-mail address: bucurileana@yahoo.com

GAVRIIL PALTINEANU

TECHNICAL UNIVERSITY OF CIVIL ENGINEERING BUCHAREST

DEPARTMENT OF MATHEMATICS AND COMPUTER SCIENCE

BD. LACUL TEI 124, SECTOR 2, 38RO-020396 BUCHAREST, ROMANIA

ORCID: 0000-0002-9274-2727

E-mail address: gavriil.paltineanu@gmail.com 